\title{
Schopenhauer y el idealismo del color
}

\author{
Eduardo Charpenel Elorduy \\ Rheinische Friedrich-Wilhelms-Universität Bonn \\ s5edchar@uni-bonn.de
}

\section{Introducción}

Las teorías científicas y filosóficas suelen tener muy diversos orígenes. Por un lado, tenemos análisis profundamente meditados que han conducido a la elaboración de grandes proyectos: encontramos ahí el paradigmático ejemplo de Kant, quien a lo largo de toda una década se limitó a concebir la monumental arquitectura de la Crítica de la razón pura. Por otro lado, vemos también que situaciones más bien azarosas o contingentes han desencadenado grandes descubrimientos: tenemos ahí el anecdótico evento según el cual Newton habría descubierto la ley de la gravitación universal a partir de que una manzana cayera sobre su cabeza, y el consabido caso de Wilhelm Röntgen, quien por accidente se percató de la penetrante radiación que los rayos catódicos ejercían a través los cuerpos, dejando tras de sí una imagen semejante a la de una fotografía: un curioso fenómeno que permitió identificar lo que hoy conocemos como rayos x. Pocas veces, sin embargo, nos topamos una «teoría por encargo»o una «teoría por conveniencia». En esta ocasión, discutiré una 
propuesta que efectivamente posee esta naturaleza: la teoría del color de Arthur Schopenhauer.

El origen de ésta es, en efecto, bastante peculiar. Schopenhauer vivía con su madre en Weimar a inicios de la década de 1810, lo cual le permitió conocer a un amplio número de figuras literarias y filosóficas que se daban cita en las tertulias intelectuales organizadas por ella. Entre todos esos personajes, sin duda, el que más resaltaba era Goethe, quien ya para ese entonces era una celebridad a lo largo y ancho de Europa. Por esas mismas fechas, Goethe había publicado su Teoría de los colores, la cual sería, a su juicio, la obra que terminaría por consagrarlo como una figura universal en el pleno sentido del término: alguien que pudiera amalgamar en su obra tanto las artes como las ciencias. Este motivo llevó al autor del Fausto a colaborar en varias revistas de corte científico y a entablar contactos con físicos, fisiólogos, biólogos y químicos. Contar con un círculo intelectual que discutiera y avalara su teoría tendría como consecuencia, según Goethe, que tarde o temprano se le diera el reconocimiento debido a su teoría, y que con ello quedara superada para siempre la óptica newtoniana.

Ése es el contexto en el cual Goethe y Schopenhauer entablan un trato mucho más próximo ${ }^{1}$. En el verano de 1813, tienen la oportunidad de reunirse de modo habitual y discuten la importancia de la teoría de los colores. Schopenhauer, por lo que sabemos, no estaba particularmente entusiasmado por esta temática, pero veía en ella una valiosa oportunidad para que el poeta hiciera las veces de padrino intelectual y lo

\footnotetext{
${ }^{1}$ En realidad ya se habrían conocido por primera vez desde 1807. Pero Goethe no parecía muy interesado en los adelantos y las aptitudes de Schopenhauer, lo cual le causaba a este último una gran mortificación. Como puede entenderse en este contexto, la teoría de los colores se presentaría posteriormente como una oportunidad única para entablar un vínculo mucho más próximo. Ver Safranski 2008; 133ss.
} 
introdujera de modo triunfal a la vida cultural europea. Ante las exhortaciones que recibe de Goethe de seguir esta línea de investigación, Schopenhauer decide tomarle la palabra, con la promesa de convalidar, desde el idealismo filosófico, todos los postulados básicos goetheanos. En 1815, se publica Sobre la vista y el color, pero el resultado es todo menos el esperado por su autor: Goethe se desentiende del escrito y trata con distancia e indiferencia a su autor, rechazando toda petición de hacerle promoción o de darle su aval público². Quizás la situación no era para menos: en éste se distanciaba de muchas tesis goetheanas y, más aún, presentaba su propuesta como una superación integral de todas las anteriores. El tenor de su discurso no hallaría eco alguno en el poeta. Schopenhauer tuvo que luchar por otros medios para alcanzar su objetivo: se dirigió a editores de publicaciones especializadas con el ofrecimiento de hacer él mismo resúmenes e incluso una traducción al latín de su escrito. Estos intentos no dieron tampoco el fruto esperado y, al final de cuentas, el reconocimiento que buscó con tanto afán sólo le llegó en su vejez con la publicación de Parerga y paralipómena, y se dio más bien gracias a su explicación, de un modo mucho menos erudito y mucho más afable, de las tesis principales de su sistema filosófico.

Sobre la visión y los colores, en cambio, ha recibido desde entonces una atención casi inexistente por parte de sus lectores y estudiosos. Este artículo tiene como propósito reparar esta carencia en el contexto hispanohablante y brindar una exposición de algunos de sus aspectos más significativos. El orden que seguiré es el siguiente: en un primer momento, ahondaré en algunos puntos generales de la doctrina de

\footnotetext{
${ }^{2}$ Para un estudio más detallado de este intercambio epistolar, remito a la traducción que realicé de la correspondencia schopenhaueriana; ver Schopenhauer 2008.
} 
Schopenhauer; en un segundo momento, expondré las que son, a su juicio, las principales actividades oculares y la escala cromática; en un tercer momento, discutiré algunas de las principales dificultades del apriorismo de los colores y una posible solución interpretativa; finalmente, me centraré en la distinción que hace Schopenhauer entre colores físicos y químicos, y en unas tesis que de modo sintético recogen los puntos principales de su postura.

No es nada menor la forma en que Schopenhauer introduce sus planteamientos dentro del contexto de la discusión sobre el color. Desde las primeras líneas, el filósofo habla, en un tono exacerbadamente autosuficiente, de cómo él será el primero en la historia en brindar una teoría del color coherente y sólida y, por lo mismo, una verdadera ciencia:

Cuando la teoría no se encuentra constantemente apoyada y fundamentada sobre hechos, resulta ser una quimera (Hirngespinst); cada experiencia singular, separada pero verdadera, tiene mucho más valor. Por otro lado, los datos singulares de una determinada periferia del territorio no constituyen una ciencia hasta que un concepto común (gemeinsamen Begriff) comprenda y contenga su esencia más íntima [...] (Schopenhauer 1986; 198. La traducción de las citas de esta obra es mía).

Los intentos anteriores, particularmente los de Goethe, tuvieron el mérito indudable de recopilar de modo ordenado datos valiosísimos para la eventual elaboración de dicha teo- 
ría ${ }^{3}$. Nótese aquí el tono tajante de la afirmación: sus antecesores pudieron hacer aseveraciones sueltas sobre distintos fenómenos y acontecimientos, pero esto sugiere de un modo bastante explícito, por el contexto general del discurso, que no pudieron comprenderlos por entero, toda vez que no contaban con una perspectiva más general o abarcante. La única «teoría» que se habría presentado en este rubro sería la de Newton, pero sería una de corte pernicioso y sofístico. La acusación que el filósofo acomete aquí recuerda claramente a la que Kant esgrimiera contra los dogmáticos metafísicos: no contar en sus discusiones con el sujeto, quien es en realidad la posibilidad misma de todo el conocimiento. Realizar todo un constructo en el que se expliquen los colores sin ninguna referencia aparente hacia quien las observa, es una tarea fatua, por no decir que absurda. El giro copernicano en relación a los colores habría, pues, de efectuarse de la siguiente manera: considerando el fenómeno de la vista desde los ojos —más en concreto, desde la retina- hacia la luz, y no viceversa. El realismo y el objetivismo, tal y como la historia sobre el pensamiento en esta materia habría mostrado, son caminos truncos que sólo conducen a aporías. Es necesario tomar al sujeto como punto de partida, tanto en un sentido trascendental como fisiológico. En un sentido trascendental, ya que todo conocimiento, para ser considerado como tal, necesita de intuiciones y conceptos. Concretamente, Schopenhauer piensa en la organización de los datos sensibles en intuiciones y su subsunción ordenada bajo el concepto de causalidad: dos tareas que garantiza de excelente forma el entendimiento ${ }^{4}$. En

\footnotetext{
${ }^{3}$ Con estas palabras califica Schopenhauer la teoría goetheana: «Son datos importantes, completos y significativos: un rico material para una futura teoría del color» $(1986 ; 197)$.

${ }^{4}$ Véase sobre este punto el siguiente ilustrativo pasaje donde Schopenhauer menciona esto
} 
un sentido fisiológico, porque el punto idóneo para elaborar una teoría del color son los ojos mismos, y no la composición física de la luz. Hay que entender la visión, pues, como un fenómeno que ocurre en el ojo, y la luz como aquello que da pie para que ese fenómeno ocurra. La visión ocurre propiamente en el ojo y no en los objetos. Claridad, oscuridad y color son, en realidad, modificaciones de la retina.

En cierta medida, podemos decir que el punto de partida es goetheano en lo esencial: por un lado, porque sigue el postulado del poeta según el cual los ojos son un instrumento mucho más apropiado para estudiar los colores que los lentes y prismas de un laboratorio; por otro, porque toma como fundamento empírico lo que éste había señalado anteriormente con relación a los «colores fisiológicos». El fenómeno que Goethe había estudiado era el de cómo, después de que nuestra vista se hubiera asentado durante mucho tiempo en la contemplación de un objeto de un color, seguiría percibiendo el mismo, incluso momentos después de ya no estarlo observando (ver Goethe 2003; 457-470). Con este simple pero ingenioso descubrimiento, Goethe y Schopenhauer pensaban dejar suficientemente en claro la jerarquía superior del sujeto con respecto al objeto dentro de la producción del color. Pero a juicio del filósofo, a su mentor le habría hecho falta dar todavía un último y decisivo paso: Goethe todavía permanece bajo una imagen «dogmática» de los colores al seguir consi-

en relación al uso de la vista: «El aprendizaje de la visión por parte de los niños y de los ciegos de nacimiento que son operados, la visión simple de lo percibido por duplicado con los dos ojos, la visión doble o el tacto doble cuando los órganos sensoriales sufren una dislocación de su lugar habitual [...] la transposición a los objetos externos de los colores [...]: todo esto supone pruebas firmes e irrefutables de que toda intuición no es sólo sensual, sino también intelectual y, por consiguiente, presupone la ley de causalidad [...]» (2003; 95). Esta postura, como se puede apreciar, iría claramente en una posición conceptualista de la organización de la experiencia, muy en la línea de filósofos como Sellars o McDowell. 
derando la mayor parte de éstos como propiedades reales de los objetos. Inspirado por el sistema kantiano, Schopenhauer afirma que el color no es más que una sensación y resulta independiente del objeto con el cual lo asociamos - por circunstancias y modificaciones de muy distinta índole, un mismo objeto puede producir una amplísima gama cromática, lo cual nos habla del color como una propiedad más bien accidental y no esencial de los objetos.

Así pues, la luz hay que interpretarla como el fenómeno que da pie a la actividad de la retina. Schopenhauer asocia el blanco con la luz y afirma que cuando ésta se hace presente, el ojo lleva a cabo su actividad de modo completo. En otras palabras, la presencia de la luz genera un máximo de intensidad con respecto a nuestras capacidades visuales. En contraste, cuando no hay luz, lo que tenemos es oscuridad (negro) y, en consecuencia, una inactividad de nuestra retina. Con la introducción de esta dicotomía, Schopenhauer rescata una intuición clásica según la cual la luminosidad sería representable en un esquema lineal, teniendo a estos dos polos como sus extremos, y al gris y otras tonalidades opacas como un punto medio. Estos puntos articulan de manera substancial el resto de su discurso y son claves para una correcta comprensión del mismo.

\section{II}

Antes de dar un paso más adelante, hay que hacer notar que Schopenhauer realiza importantes distinciones sobre las actividades oculares: 1) divisibilidad intensiva de la retina: la variación en la afección de la intensidad de la luz sobre nuestros ojos; 2) divisibilidad extensiva de la retina: la retina tiene pequeñísimas pero múltiples partes, lo cual permite que cuan- 
do contemplemos un amplio panorama cromático, ésta sea afectada por distintos frentes y captemos una diversidad de colores dentro de una misma imagen. Sin embargo, la distinción que más le interesa señalar es: 3) divisibilidad cualitativa de la retina: cuando contemplamos por un prolongado tiempo un objeto oscuro, nuestra retina se cansa y ya no reacciona de la misma forma ante los estímulos de un objeto brillante. Lo que ocurre en ese caso, más bien, es que contemplamos una mancha o un espectro (Spektrum) sobre la superficie del nuevo objeto en cuestión (ver Schopenhauer 1986; 224). Este fenómeno es el que solemos experimentar con frecuencia en la vida cotidiana, por ejemplo, cuando después de estar por mucho tiempo en un lugar oscuro salimos a la calle y vemos con dificultad nuestro entorno. También ocurre lo mismo de modo inverso: cuando estamos en un lugar luminoso e ingresamos a uno oscuro, vemos varias manchas luminosas a nuestro alrededor, y nuestra vista tarda un pequeño lapso de tiempo en establecer un ajuste.

A partir de esta experiencia común, Schopenhauer plantea una nueva escala cromática. Como habíamos dicho, el blanco y el negro se encuentran en los extremos opuestos: un planteamiento que, en realidad, se había gestado dentro de la antigüedad. La originalidad de su teoría estriba más bien en que establece una proporcionalidad matemática entre los otros colores fundamentales de su escala: el violeta, el azul, el verde, el rojo, el naranja y el amarillo.

Schopenhauer diseñó su escala cromática de modo que la suma del color y su color opuesto siempre da uno como resultado. El planteamiento del cual parte para decir esto es que la actividad completa de la retina necesariamente produce el color blanco. Otra forma de expresar esta idea es que en la visión de otros colores la retina no trabaja por entero, 
sino sólo en algunas de sus partes. Supongamos, pues, que uno está observando el color verde por un periodo considerable de tiempo: en esa actividad sólo está trabajando nuestra retina a la mitad de su capacidad real. En consonancia con la distinción sobre la divisibilidad extensiva de la retina, podríamos decir que se trata de un color que sólo estimula una parte de la misma. Si después volteamos nuestra mirada hacia un objeto rojo, el espectro resultante será blanco, dado que la parte de la retina inactiva entraría en juego y esta combinación conduciría a la plena actualización de su potencia. Lo mismo ocurriría en los casos donde apreciáramos el violeta y el amarillo o el azul y el naranja. Con esta concepción del blanco en términos de pares de tonalidad, Schopenhauer intenta corregir la equivocada opinión newtoniana según la cual la suma de todos los colores primarios da como resultado siete. El error, en este caso, estribaría en el siguiente punto: si sólo la combinación de dos colores puede producir el blanco, la consecuencia lógica de ello es que hablar de un número impar en el contexto de la totalidad de los colores primarios es desacertado. Esto deja ver claramente por qué para el filósofo las explicaciones clásicas serían más pertinentes que las modernas: una explicación fundamentalmente cualitativa en términos de pares o contrarios da mayor cuenta que la explicación matematizante y dogmática que no toma en cuenta al sujeto del conocimiento en cuestión ${ }^{5}$. La ventaja extra que ade-

\footnotetext{
${ }^{5}$ Un planteamiento que sin duda influye en la investigación de Schopenhauer es la concepción aristotélica de los contrarios. Véase la afinidad entre el planteamiento del Estagirita en la Física y el que estamos exponiendo: «Así pues, es manifiesto que de una u otra manera todos consideran los contrarios como principios. Y con razón, pues es necesario que los principios no provengan unos de otros, ni de otras cosas, sino que de ellos provengan todas las cosas. Ahora los primeros contrarios poseen estos atributos: no provienen de otras cosas, porque son primeros, ni tampoco unos de otros, porque son contrarios» (1888a 25-30).
} 
más tendría la teoría de Schopenhauer sobre las antiguas es su concepción crítica del sujeto.

Ahora bien, con relación a lo que se ha dicho anteriormente, cabe por supuesto hacerse una pregunta muy pertinente: ¿por qué Schopenhauer introduce estos colores y no otros? La respuesta que da es que estos colores son muestras de la actividad de la retina en sus fracciones o proporciones más elementales. De igual forma que en las escalas musicales, hay muchísimas tonalidades intermedias entre un color y otro: unas de éstas son tan sutiles que apenas serían distinguibles unas con respecto de otras (de ahí la dificultad de diferenciar entre ciertos matices, e incluso muchas veces de asignarles un nombre). Lo más conveniente resulta centrarnos en las actividades ejemplares y más claramente distinguibles, y estos seis colores son la expresión más fidedigna que tenemos de ellas. En otros términos, podríamos decir que el color es un tipo de actividad particular que tienen nuestros ojos ante la presencia de la luz y su reflejo particular sobre ciertos objetos. Aquí incluso es preciso hacer una distinción: el blanco y el negro no son colores en un estricto sentido del término, pues «Debido a que no representan ningunas fracciones, es decir, divisiones cualitativas, el blanco y el negro no son en realidad colores, como siempre se ha dicho que son» (Schopenhauer 1986; 234). Son sólo modos de indicar, con respecto a ciertos objetos, que nuestra retina se encuentra o no realizando la actividad que le es propia. Por supuesto, se trata de un uso coloquial y sumamente extendido, el de referirnos al blanco y al negro como si fueran colores. Schopenhauer en ningún momento sugiere que debamos de hablar así en un contexto cotidiano. En el ámbito intelectual y científico, sin embargo, sí es preciso tener en cuenta esta distinción y considerar que, propiamente hablando, el color sólo se predica cuando aludimos a una mayor o menor actividad 
por parte del ojo, y no así cuando nos referimos a su plena actividad o a su ausencia.

\section{III}

Schopenhauer afirmará insistentemente a lo largo de esta obra que nuestra retina tiende teleológicamente hacia la actividad de contemplar la luz 6 . La naturaleza, de un modo sumamente previsor, nos ha dotado de un órgano para situarnos en nuestro entorno gracias a la luminosidad proveniente de ciertos cuerpos. Lo que ocurre, sin embargo, es que hay medios a través de los cuales ésta no se manifiesta de la misma forma ante nuestros ojos. También existen objetos cuya superficie refracta la luz de un modo particular que sólo estimula determinadas partes de nuestra retina. En esto se ve un intento - cuyo calificativo quizás podría ser el de «romántico»— de tratar de dar una explicación en términos radicalmente opuestos a los del materialismo, el cual busca hacer una lectura integral de la naturaleza exclusivamente en términos físicos y químicos. Esta teoría schopenhaueriana, por el contrario, privilegia lo subjetivo sobre lo objetivo: un rasgo característico, según el filósofo, de las explicaciones más acertadas en relación al color a lo largo de la tradición 7 . Es por ello que la luz es concebida

\footnotetext{
${ }^{6}$ Postura que también sostiene Goethe: «Wenn wir die Augen innerhalb eines ganz finstern Raums offen halten, so wird uns ein gewisser Mangel empfindbar. Das Organ ist sich selbst überlassen, es zieht sich in sich selbst zurück, ihm fehlt jene reizende befriedigende Berührung, durch die es mit der äußern Welt verbunden und zum Ganzen wird» (2003; 462). ${ }^{7}$ Véase, por ejemplo, la caracterización de cualidades secundarias como aquellas que se dan propiamente en el sujeto: «Secondly, such qualities, which in truth are nothing in the objects themselves, but powers to produce various sensations in us by their primary qualities, i.e. by the bulk, figure, texture and motion of their insensible parts, as colors, sounds, tastes, etc.» (Locke 1998; 135).
} 
por él como un principio vital más que como un fenómeno reductible a ondas mecánicas. Por lo mismo, desarrolla un planteamiento que intenta ir mucho más allá de una descripción externa de la constitución del mundo.

Otro momento donde este subjetivismo se puede apreciar con gran claridad es cuando Schopenhauer hace la sorprendente afirmación de que poseemos un conocimiento a priori de estos seis colores fundamentales. El filósofo llega a afirmar que hombres de todos tiempos y culturas poseen un conocimiento bastante objetivo sobre los seis colores de su escala y, por ende, llama a los mismos «normas», «ideales» e incluso «anticipaciones epicúreas» ${ }^{8}$. Esta aseveración es un tanto desconcertante si tomamos en consideración, por ejemplo, los márgenes claramente establecidos por Kant en su filosofía crítica: tenemos $a$ priori formas puras de la sensibilidad y categorías que permiten ordenar la experiencia sensible, pero de ninguna forma contamos a priori con datos concretos y específicos sobre la constitución del mundo físico. Recordemos también la drástica posición humeana: las impresiones simples son irreductibles a otras más básicas y nos es imposible procurarnos esos datos fundamentales si nuestros sentidos se encuentran dañados9.

Siendo Schopenhauer un atento lector tanto de Hume como de Kant, parece muy raro que desatendiera o que hicie-

\footnotetext{
${ }^{8}$ Schopenhauer toma este nombre de Cicerón. Reproduzco la cita que él introduce en su escrito: «Una anticipación — prolepsis, tal como Epicuro la llamó- es una concepción de la cosa formada en la consciencia, sin la cual la consciencia no podría reconocerla, preguntar por ella o investigarla» (Cicerón, De natura deorum, I, 16; en Schopenhauer 1986, 232).

${ }_{9}$ «Siempre que, por accidente, las facultades que dan origen a impresiones de algún tipo se ven impedidas en sus operaciones (como le ocurre al que ha nacido ciego o sordo), no sólo se pierden las impresiones, sino también las ideas correspondientes, de modo que jamás aparecerá en la mente el menor rastro de unas u otras. Y esto es verdad no sólo en el caso en que los órganos sensoriales estén completamente destruidos, sino también cuando no han sido puestos en funcionamiento para producir una impresión determinada. No podemos hacernos una idea correcta del sabor de una piña sin haberla probado realmente» (Hume 2008; 6. Cito aquí la paginación crítica de Selby Bigge y no la de la propia traducción).
} 
ra caso omiso de estos postulados tan fundamentales. Me parece que ése no es el caso en este escrito que estamos analizando. Creo que una lectura que atiende a la coherencia integral de su planteamiento tiene que sostener una interpretación flexible de este pasaje: Schopenhauer no parece estar diciendo que un ciego de nacimiento pudiera tener una representación, por más elemental que sea, de estos colores. En este sentido, me parece que la postura de nuestro autor debe leerse de la siguiente forma: casi nunca encontramos una manifestación pura de estos colores dentro de la propia naturaleza. Lo curioso de esto es que nosotros podemos identificar casos en los que el naranja de un objeto tiende más hacia el amarrillo o el rojo, o casos en los que el azul tiende más hacia el violeta o el verde. Ahora bien, ¿en función de qué podemos hacer esta distinción? Parece absurdo decir que nuestra noción de rojo haya sido adoptada a partir de la primera vez que vimos un objeto rojo en toda su pureza. Pienso que, a juicio de Schopenhauer, la postulación de un reconocimiento inmaculado de cada uno de estos seis colores sería algo completamente infundado y ridículo. Lo que habría que decir, más bien, es que nosotros tenemos la capacidad de pronunciarnos sobre la pureza o impureza de una tonalidad -me refiero con esto a establecer la proximidad de un color a otro en la experiencia-, dado que nosotros podemos reconocer, por la constitución particular de nuestro aparato visual, cuándo una tonalidad genera la actividad de la retina correspondiente, en términos de los patrones matemáticos de los que anteriormente hemos hablado.

Con esto no quiero suscitar un equívoco: no es mi intención afirmar que nosotros hagamos conscientemente un cálculo matemático para determinar el grado de actividad de nuestra retina. Me siento más inclinado a pensar lo siguiente: nuestra retina opera ya por naturaleza conforme a estos pa- 
rámetros matemáticos, como si estas fracciones de su propia actividad fueran criterios objetivos a partir de los cuales uno puede juzgar por naturaleza la pureza o impureza en el color de los objetos empíricos. En otros términos, estas fracciones en su actividad se convierten en paradigmas intersubjetivos que nos sirven como base para poder distinguir unos y otros colores. Quizás lo que puede provocar cierta confusión es que Schopenhauer haga en este punto en concreto una comparación con la geometría: el filósofo afirma que nunca nadie ha visto un triángulo o un cuadrado perfecto en la realidad, pero que contamos con los suficientes elementos a priori para poder representárnoslo independientemente de la experiencia y después trasladar ese conocimiento a un cálculo sobre lo empírico. Me parece que lo que habría que decir aquí es que la analogía es ilustrativa, pero que presenta límites bastante bien definidos: si bien en el caso de la geometría contamos con criterios a priori en la representación de estos cuerpos, éste es un criterio de orden trascendental, toda vez que el espacio es la forma pura a la que apelamos para figurarnos los mismos. En cambio, en lo que atañe a la vista y a estos seis colores, lo que Schopenhauer postula tendría que ver más con la disposición, la constitución y la receptividad mismas de nuestro aparato visual, y no así con una condición trascendental de nuestro conocimiento. De ahí que el a priori sería en este caso una constitución integral de la estructura y el arreglo mismo de nuestra anatomía. Me parece que este «a priori fisiológico» es, sin dudas, un claro signo de la originalidad de la propuesta de Schopenhauer.

Con lo anterior, queda claro por qué para el filósofo un ciego de nacimiento no tendría un conocimiento de estos colores, a pesar de que éstos, en un sentido muy preciso, sean a priori: la razón de ello consiste en que sus órganos se en- 
cuentran dañados y nunca ha podido contemplar un objeto y juzgarlo con estos criterios. Además, dicho sea de paso, esta postura puede explicar con gran facilidad el daltonismo: ciñéndonos férreamente a la teoría schopenhaueriana, tendríamos que decir que Newton sería incapaz de dar cuenta fehaciente de un fenómeno como éste, toda vez que considera a los colores como una propiedad objetiva de la realidad y no como modificaciones del mismo ojo ${ }^{10}$.

\section{IV}

En el penúltimo parágrafo de su obra, Schopenhauer establece una distinción entre colores físicos y químicos. Esta distinción, en realidad, no es original de él: varias teorías sobre los colores, incluyendo la del mismo Goethe, apelaban a esta categorización y desarrollaban muchos de sus más importantes conceptos en función de la misma. Lo que resulta curioso aquí, sin embargo, no es tanto la introducción misma de esta distinción, sino la particular exposición que hace de ella el filósofo a la luz del idealismo.

En términos generales, los colores físicos son sólo tonalidades que se muestran dentro de circunstancias específicas relacionadas con la combinación de la luz y la transparencia del medio: circunstancias que, por su misma condición, son cambiantes y mudables. Así pues, podemos averiguar las características de cierto entorno luminoso y estudiar la co-

\footnotetext{
${ }^{10}$ Curiosamente, Schopenhauer también creía que una confirmación de su teoría era también el hecho de que las fotografías o daguerrotipos de la época sólo pudieran captar las figuras y no los colores de las cosas. El hecho de que las fotografías fueran en blanco y negro comprobaría que el color no está en la realidad sino en el ojo que observa. Ver Schopenhauer 1986; 266.
} 
nexión de leyes y regularidades que producen determinadas condiciones cromáticas. A pesar de que no hayamos hecho la investigación en cuestión, resulta claro que nuestro intelecto vincula, de modo necesario, la producción de esas condiciones a unas causas y fenómenos específicos: en este caso, la condición a la que se remite es a la luz y a la transparencia del medio. Independientemente de los detalles particulares que podemos averiguar gracias a una investigación empírica, sabemos que la percepción cambiante sobre el color de un mismo objeto nos conduce irremisiblemente a la influencia de la luz. Podemos decir, entonces, que tenemos claramente definido cuál es el origen de una determinada percepción. En consonancia con su tradición filosófica kantiana, pero yendo más allá de la misma en modo innovador, Schopenhauer vuelve a identificar esa necesidad con un cierto tipo de a priori; en esta ocasión, sin embargo, con relación a la causa a la que acudimos para apreciar el brillo o la opacidad de ciertos colores.

En contraparte, lo que sucede con los colores químicos es una absoluta y gran interrogante: no tenemos elementos para determinar por qué ciertos colores son inherentes a ciertos objetos. Permanecemos ante una especie de incógnita al tratar de saber por qué los objetos naturales poseen tal o cual color. Es mucho más fácil saber por qué la pradera se ve gris y nebulosa en el alba que saber por qué las manzanas son rojas o por qué los cuervos son negros; parece tan caprichoso que manzanas y cuervos tengan tales colores que como si tuvieran los colores contrarios. Aunque Schopenhauer no posee ninguna herramienta - por ejemplo, un artefacto tan sofisticado como los modernos espectroscopios- para determinar por qué se da esa producción de forma natural, lo que es un hecho en su opinión es que si, en un momento dado, las leyes de la producción de esos objetos fueran descubiertas, éstas serían 
necesariamente empíricas y, por lo mismo, sujetas a cualquier contingencia y variabilidad. A tal punto llega la perplejidad de Schopenhauer en esta materia, que no duda en llamar a los colores físicos y químicos comprensibles (verständliche) e incomprensibles (unverständliche) respectivamente (ver 1986; 269).

Como se puede suponer, Schopenhauer ahonda en la última parte de su obra mucho más sobre los colores físicos, ya que hay mucho mayor material para teorizar en relación al fenómeno mismo de la luz. Hay ciertos fenómenos que trae a colación en este momento para redondear de mejor forma su propuesta. Menciona cinco tesis fundamentales sobre los mismos: estas tesis, en realidad, son en cierta forma una síntesis general de su propia teoría. Haré una breve explicación de todas a modo de corolario de nuestra exposición sobre la materia.

1. Sólo los estímulos externos pueden causar color. Cuando recibimos un estímulo, se dan una serie de divisiones cualitativas y cuantitativas en el ojo. De ninguna forma se encuentran los colores de modos independientes fuera de nuestra retina. La única posible reconciliación entre la teoría de Newton y la de él sería la armonía preestablecida; es decir, la teoría según la cual lo mismo que el sujeto percibe es exactamente lo mismo que existe en la realidad, de modo que posiciones como el realismo o el idealismo serían equivalentes. Hacer tal sugerencia sería puro dogmatismo, toda vez que afirmaciones así requieren traspasar por mucho los límites de nuestro conocimiento.

2. Cada color es la mitad cualitativa de la actividad total de la retina y, sólo otro color puede completar la mitad restante. De esto se sigue que los colores se presenten en pares dentro de la naturaleza y de ninguna forma de modo aislado. Por ende, postular una escala cromática de siete colores (un número impar) es un total desacierto. 
3. El número de colores existentes es infinito. Mediante la división del círculo de los seis colores antes descritos puede surgir cualquier color. Lo que ocurre en realidad es que nosotros identificamos con facilidad tres pares distintos, ya que la actividad de la retina se divide proporcionalmente en números simples.

4. La causa externa del color - un estímulo- es modificable en la misma medida que lo es la retina. Esto plantea la posibilidad de una grandísima cantidad de colores y no sólo de siete, como sería a juicio de Schopenhauer, la conclusión de la propuesta newtoniana.

5. En la retina, el color siempre está entremezclado con una oscuridad o sombra (skerión, es el término griego que el filósofo emplea). La causa externa del color es una luz disminuida que imparte cierta intensidad que deja sin actividad a una parte matemáticamente proporcional de la retina. El eje de la explicación, en suma, es orgánico y no físico.

El interés por la propuesta schopenhaueriana, como he mencionado, quedó por mucho tiempo desatendido $\mathrm{y}$, hasta la fecha, es un material muy fértil de investigación. Por supuesto, en términos sistemáticos, hay mucho que podría criticársele a la luz de varios desarrollos contemporáneos: piénsese, por ejemplo, en los rayos infrarrojos. Éstos son vibraciones de onda claramente existentes, parte integral del prisma cromático, que por su índole específica, los seres humanos no podemos ver sin la ayuda de aparatos especiales. ¿Significa esto, entonces, de acuerdo con el cuarto punto del filósofo antes citado, que dichos colores no existen porque no afecta de modo significativo a la divisibilidad cualitativa de la retina? Parece que, ante casos de esa naturaleza, sostener un idealismo radical del color como el de Schopenhauer difícilmente es sostenible hoy en día. A pesar de ello, considero que podría 
darse con esta teoría algo parecido a lo que ocurrió con la de Goethe: a su Teoría de los colores durante mucho tiempo se le vilipendió y se le consideró una ensoñación romántica del poeta, pero a últimas fechas ha recibido una atención inu-sitada por gran cantidad de estudiosos, tanto de físicos como de psicólogos y filósofos de la mente. Los intentos por situar con claridad las propuestas de Newton y Goethe a la física y a las ciencias cognitivas, respectivamente, han ayudado bastante para comprender los alcances reales de sus aportaciones. Los estudios que procuren hacer lo mismo con Schopenhauer seguramente podrán obtener beneficios semejantes.

\section{BIBLIOGRAFÍA}

Aristóteles. (1995). Física. Tr. Guillermo R. de Echandía. Madrid: Gredos.

Goethe, Johann Wolfgang von. (2003). Naturwissenschaftliche Schriften, Hamburger Ausgabe, Bd. XIV. Hamburg: C.H. Beck.

Hume, David. (2008). Tratado de la naturaleza humana. Tr. Félix Duque. Madrid: Tecnos.

Locke, John. (1998). An Essay Concerning Human Understanding. London: Penguin.

Safranski, Rüdiger. (2008). Schopenhauer y los años salvajes de la filosofía. Tr. José Planells. México: Tusquets.

Schopenhauer, Arthur. (2008). Cartas desde la obstinación. Tr. Eduardo Charpenel. México: Los libros de Homero.

Schopenhauer, Arthur. (2003). El mundo como voluntad y representación. Tr. Roberto R. Aramayo. Madrid: FCE.

Schopenhauer, Arthur. (1986). Kleinere Schriften. Frankfurt: Suhrkamp. 


\section{RESUMEN}

En 1810, Goethe publicó su tratado titulado Zur Farbenlehre (Teoría de los colores). Con la intención de respaldar los postulados básicos de la teoría de Goethe y con ello ganar el apadrinazgo del escritor, en 1815 Schopenhauer redactó Über das Sehn und die Farben (Sobre la visión y los colores). La teoría schopenhaueriana de los colores, tal como explica el autor, fue desatendida por mucho tiempo a pesar de que en ella hay material sumamente valioso. Schopenhauer logra articular su teoría de la visión con una descripción idealista del color. Creer que los colores son propiedades reales de los objetos es una postura, según Schopenhauer, dogmática: el color es una sensación que se explica a partir de que la luz permite la actividad de la retina. Tras un análisis detallado de la concepción schopenhaueriana del color, el autor apunta sus atinos y desatinos.

Palabras clave: Schopenhauer; Goethe; color; visión; idealismo.

\section{ABSTRACT}

In 1810, Goethe published his treatise entitled Theory of Colors. In order to support the basic tenets of Goethe's theory and gain the sponsorship of this thinker, Schopenhauer wrote in 1815 On Vision and Colors. Schopenhauer's theory of colors, as the author explains, was neglected for a long time despite its extremely valuable material. Schopenhauer articulates his theory of vision with an idealist description of color. According to Schopenhauer, believing that colors are real properties of objects is a dogmatic position: color is a sensation that is explained by the light that allows the activity of the retina. After a detailed analy- 
sis of Schopenhauer's conception of color, the author points out its virtues and blunders.

Key words: Schopenhauer; Goethe; color; vision; Idealism. 
